\title{
Dinámicas sociales en la década del 90: trayectoria laboral petrolera en el norte de Santa Cruz
}

\section{Social dynamics in the 90s: labor trayectory of petroleum in the north of Santa Cruz}

\author{
Pamela Soledad Leopardo, Dina Rozas \\ pame_eva02@hotmail.com \\ Universidad Nacional de la Patagonia Austral
}

Recibido: 01/06/2020. Aceptado: 17/11/2020

\section{RESUMEN}

Santa Cruz, es una provincia caracterizada por el fuerte impacto laboral de las empresas petroleras en la década del 90. Esto permitió el crecimiento de la población por medio de la migración de mano de obra proveniente de otras provincias. Pico Truncado y Caleta Olivia son dos de las localidades situadas al norte de la provincia que crecieron a raíz de la demanda laboral.

En el siguiente informe se abordará el testimonio de uno de los trabajadores de Pico Truncado, Francisco Jaramillo y las trayectorias y herramientas utilizadas que configuraron nuevas formas de aprendizaje desde una temprana edad. Es por ello, que el objetivo principal de este informe es aportar a la construcción de la dinámica social de la década del 90 en Santa Cruz, a través del testimonio de un ex empleado petrolero, identificando el contexto, los procesos y las herramientas empleadas por las empresas petroleras para incorporar nuevos trabajadores.

El objetivo de este informe está vinculado a dos de los objetivos específicos del proyecto de investigación "Cartografía de los '90: Cultura y Política de Santa Cruz: "Identificar aspectos/dinámicas particulares que caracterizaron la dinámica social de Santa Cruz en la década de los '90 “y "Caracterizar los sujetos sociales y sus grupos de referencias".

De esta manera, utilizando como herramientas la entrevista con la cámara como una herramienta de archivo audiovisual y análisis a partir del proceso de investigación dentro del Gabinete de Experiencias Pedagógicas: Pensamiento y Habla, se intenta aportar en la reconstrucción de la dinámica social de Santa Cruz en la década del 90.

Palabras clave: dinámica social; trayectorias; trabajadores; empresas petroleras.

\begin{abstract}
Santa Cruz is a province characterized by the strong labor impact of the oil companies in the 90's. This allowed the population to grow through the migration of labor from other provinces. Pico Truncado and Caleta Olivia are two of the towns located in the north of the province that grew as a result of labor demand.

The following report will address the testimony of one of the Pico Truncado workers, Francisco Jaramillo and the trajectories and tools used that shaped new forms of learning from an early age. For this reason, the main objective of this report is to contribute to the construction of the social dynamics of the 1990s in Santa Cruz, through the testimony of a former oil employee, identifying the context, processes and tools used by oil companies to incorporate new workers.
\end{abstract}


The objective of this report is linked to two of the specific objectives of the research project "Cartography of the 1990s: Culture and Politics of Santa Cruz: "Identify particular aspects / dynamics that characterized the social dynamics of Santa Cruz in the decade of the '90 "and" Characterize social subjects and their groups of references ".

In this way, using the interview recorded with the camera as a tool for audiovisual archiving and analysis based on the research process within the Cabinet of Pedagogical Experiences: Thought and Speech, an attempt is made to contribute to the reconstruction of the social dynamics of Santa Cruz in the 90's, as well as the new structures and settings.

Keywords: social dynamics; trajectories; workers; oil companies.

\section{INTRODUCCION}

Este trabajo se inscribe en el proyecto de investigación "Cartografía de los '90: Cultura y Política de Santa Cruz", orientado a construir el pasado reciente en la provincia desde esas dimensiones en esa década. Y de manera específica, aporta a los objetivos específicos del proyecto de investigación mencionado, entre otros, de "Identificar aspectos/dinámicas particulares que caracterizaron la dinámica social de Santa Cruz en la década de los '90 “y "Caracterizar los sujetos sociales y sus grupos de referencias".

En ese marco el objetivo de este informe es aportar a la construcción de la dinámica social a través del testimonio de un ex-trabajador petrolero en la localidad de Pico Truncado, Francisco Jaramillo. La reconstrucción de las trayectorias de vida en sus diferentes dimensiones, y en este caso, vinculado a la actividad laboral, contribuyó a conocer aspectos y dinámicas que caracterizaron la dinámica social, así como a los sujetos sociales y sus grupos de referencia. La actividad extractiva vinculada con el petróleo en el Sur de la Patagonia formó empresas que impulsaron la demanda laboral. Esta demanda contribuyó a la configuración de una estructura y nueva división de trabajo innovadora. Esta configuración de las prácticas laborales se caracterizó por un proceso de aprendizaje basado en el oficio como herramienta principal.

La década del 90 en Santa Cruz estuvo atravesada en la escena social, económica y política por acontecimientos que reflejaron la situación de crisis que atravesaba Argentina a nivel nacional en esa década, como fue el caso de la privatización de Yacimientos Petrolíferos Fiscales S. A. (YPF S. A.), la restructuración de las empresas, la quiebra de algunas, la configuración de horario de las jornadas laborales, los paros sindicales, el impulso de nuevas instituciones educativas que permitían nuevas formas de institucionalizar los oficios, como las escuelas secundarias técnicas e industriales.

Entre los sujetos sociales del noroeste de la provincia se encontraban los trabajadores que venían desde afuera para ocupar los oficios y los trabajos en las empresas petroleras que se iniciaban en la década del 80 . Y con ello la expectativa de reconstruir una nueva forma de vida, una nueva estructura de familia y una interculturalidad entre provincias.

En ese sentido, en el abordaje del testimonio de Francisco Jaramillo se focalizará en las trayectorias y herramientas utilizadas en su trabajo, como nuevas formas de aprendizaje desde una temprana edad. Validadas por las empresas petroleras para incorporar nuevos trabajadores, a la vez que configuraron nuevas estructuras, escenarios y dinámicas sociales desde lo laboral.

En cuanto al aspecto metodológico, la entrevista en profundidad y la cámara como una herramienta de archivo audiovisual y de registro, tomo su rol al involucrar la imagen del entrevistado a través de la construcción del plano y del encuadre. Ello sumado al análisis de la información obtenida que se llevó a cabo en el Gabinete de Experiencias Pedagógicas: 
Pensamiento y Habla, posibilitó la producción del testimonio de Jaramillo como objeto de abordaje, la reconstrucción de su trayectoria de vida y su análisis.

\section{MARCO DE REFERENCIA}

La Unidad Académica Caleta Olivia (UACO) de La Universidad Nacional de la Patagonia Austral (UNPA), en conjunto con la Universidad Nacional San Juan Bosco (UNSJB), reúne varios docentes-investigadores que han trabajado a lo largo de los años sobre la temática laboral de los trabajadores petroleros en las provincias de Santa Cruz y Chubut. Entre ellos, se encuentran Daniel Cabral Márquez, Julia Alcaín, Lía Guerra, Natalia Barrionuevo y Hernán Palermo. En el libro "El mundo del trabajo petrolero en la cuenca del Golfo San Jorge. Estudios sobre el pasado y el presente", el autor Gabriel Carrizo hace una compilación de los investigadores que estudian sobre el tema. El libro configura parte de la historia del petróleo que va desde el abordaje de la figura de Mosconi, a través de las prácticas, usos y disputas en el ritual fúnebre desarrollado por Gabriel Carrizo, seguido de los aportes sobre el General Enrique Mosconi como primer presidente de los Yacimientos Petrolíferos Fiscales (YPF), cómo configuró la importancia de YPF en el Golfo San Jorge entre los habitantes de la región, investigación de Daniel Cabral Marquéz. Asimismo, las investigaciones de Julia Alcaín y Lía Guerra abarca a los trabajadores que quieren ingresar al mercado laboral petrolero y los que están insertos en él, enfocando en sus trayectorias y subjetividades, tomando como referencia a los trabajadores de la Empresa Patagónica Sociedad Anónima (EMPASA). Natalia Barrionuevo investiga sobre las desigualdades sociales en torno al género femenino en las empresas petroleras con mayor índice de trabajadores masculinos en la localidad de Comodoro Rivadavia, Chubut. Y, por último, Hernán Palermo, investiga sobre la violencia laboral naturalizada entre los trabajadores petroleros de Comodoro Rivadavia.

Otras investigaciones relevantes son las realizadas por Gabriel Carrizo, docente investigador de la UNSJB. Una de ellas es la que presenta en el artículo "De la invisibilización a la exaltación. Mosconi, YPF y el petróleo en el discurso kirchnerista". La investigación se centra también en este artículo en la reconstrucción historia del General Enrique Mosconi como símbolo de la industria petrolera y nacionalismo frente a la privatización de YPF en la década del 90' en la localidad de Comodoro Rivadavia. "De militar autoritario a héroe del nacionalismo petrolero. Acerca de los usos políticos de Mosconi en la Argentina contemporánea", sigue la línea de destacar como símbolo nacional a el General Enrique Mosconi y sus aportes a la industria petrolera y los usos políticos que se le han dado a su figura hasta la actualidad.

Además, en la UNPA se propició la realización de una investigación coordinada por Agustín Salvia denominada "La Patagonia de los noventa. Sectores que ganan, sociedades que pierden", esta estuvo centrada en el desarrollo económico de la región y aporta una exhaustiva investigación sobre el proceso de privatización de YPF e YCF.

El tema de este informe concierne a lo laboral en la actividad petrolera. El testimonio de Francisco Jaramillo aporta a conocer aspectos de la dinámica social de un sector de la sociedad situado al Noroeste de la provincia, el sector petrolero, por lo que se consideraron pertinentes las investigaciones mencionadas. Lo importante de este testimonio es la unión de dos provincias, Santa Cruz y Chubut, logrando así, hacer el recorrido de un trabajador de petróleo de la década del 90.

\section{Marco conceptual}

En lo que refiere al tema que consiste este informe, se reconoce como marco conceptual el que aborda al neoliberalismo como corriente política y económica. Esta corriente tiene 
características fundamentadas en la liberalización de la economía, el libre comercio, la reducción del gasto público y principalmente, la intervención del Estado en la economía en favor del sector privado. En argentina, el neoliberalismo y las privatizaciones de las empresas empezaron a gestarse a fines de la década del 80, potencialmente en 1987, bajo el Gobierno de Raúl Alfonsín. Al asumir Carlos Menem en julio de 1989, se sancionó la Ley 23.696, conocida como la Ley de Reforma del Estado, que fue lo que permitió la privatización de más de 60 empresas estatales, algunas de servicios esenciales como el abastecimiento de agua, gas, electricidad y transporte.

La Mg. Dina Rozas y la Lic. María Florencia Navarro en el informe final correspondiente al PI 29/D079 "Cartografías de los 90. Cultura y política en Santa Cruz" mencionan: "La instalación de un modelo de política neoliberal que, no solo se implementó en el área de la economía, sino que impregnó el conjunto de políticas públicas producidas por el gobierno menemista, de entre las cuales cobraron centralidad las políticas de privatización de diferentes organismos dependientes del Estado (empresas de producción y de servicios, ej. Empresa Nacional de Telecomunicaciones, Aerolíneas Argentinas, YPF, de activos, etc.) en el marco de un proceso de "achicamiento" del Estado y del gasto público. Es decir, dotar de "mayor eficiencia" la gestión del Estado y sus instituciones".

Además, la década del 90 constituye una escena de trayectorias laborales enmarcadas en la migración y en el trabajo no institucionalizado. De 1991 al 2001 Santa Cruz creció un 23\%, (Rozas, Dina. Navarro, Florencia. Informe Final PI29/D079. Pag.9). Sin embargo, las empresas petroleras formaban su equipo de trabajo en las décadas del 70 y 80.

Agustín Salvia en "La Patagonia de los noventa. Sectores que ganan, sociedades que pierden" dice:
"El nuevo ordenamiento económico surgido en los noventa ha abierto nuevas formas de competencia y gestión de las relaciones económicas y laborales. Este nuevo orden económico parece estructurar un nuevo tipo de "división espacial del trabajo", a partir del cual asumen renovado protagonismo tanto los intereses de los grupos "oligopólicos" multinacionales, como los actores y conflictos estatales-locales de gestión de las relaciones laborales y de mercado." (Salvia, 1999, pág. 3.)

El presente informe aborda el testimonio de Francisco Jaramillo, enfocándose en su trayectoria laboral, lo que involucra la división de trabajo que se configuraba en las décadas del 80 y 90.

La división de trabajo, como concepto fundado por Adam Smith, consiste en la especialización y la cooperación de las fuerzas laborales con el fin de aumentar la productividad ahorrando tiempo y capital. Una de las características principales es el aprendizaje a través de la experiencia. Los trabajadores petroleros no institucionalizados aprendían por oficio con el complemento de sus pares, como el caso de Francisco Jaramillo. Ellos aprendían a través de las enseñanzas de sus superiores. Las estructuras sociales y la división de trabajo se constituyeron con la demanda de las empresas públicas y privadas, la formación de diferentes sectores que necesitaban de mano de obra. De esta manera, buscaban hombres adultos con capacidades y oficios que pudieran ejercer y aprender a través del trabajo forzado. El proceso de aprendizaje era a través de los oficios en relación con la ayuda que le propiciaba su relación con sus pares. Aprendían en base al compañerismo y buena conducta, poniendo en relevancia que los que respetaban las normas y mandatos establecidos eran los más beneficiados. Francisco: "Nosotros éramos un equipo, nos ayudábamos entre todos para aprender". 
Francisco Jaramillo es actualmente un hombre de 69 años proveniente de la localidad de Sarmiento, Chubut. Actualmente reside en la localidad de Pico Truncado, Santa Cruz. Su llegada, en la década del 80, fue a raíz de la reorganización del trabajo que se empezó a gestar en la provincia a través de las empresas petroleras. Con una trayectoria de 42 años trabajando en diferentes empresas petroleras se jubiló como oficial mecánico de motores en petróleo.

La precariedad de las provincias de la Patagonia como Chubut y Santa Cruz determinó que las personas aprendieran oficios de diferentes índoles, como mecánica y cocina desde muy jóvenes, sosteniéndose desde la base de progreso y crecimiento. La mecánica y el trabajo con los motores era determinante, los ingenieros en la zona eran pocos, de esta manera buscaban a quienes pudieran cubrir esos puestos de trabajo. Así fue como Francisco Jaramillo arribó a Pico Truncado: "Sabia de mecánica, tenía buenas referencias, era joven con energía y buena salud, tenía ganas de trabajar, de progresar y crecer". La situación del trabajo era difícil, la obtención de un lugar dentro de una empresa estaba determinada por la capacidad de la persona, sino "se aprendía a los golpes, pero siempre aprendiendo para conseguir un lugar". "Aprendí hablando con los ingenieros, empecé con la voluntad de ayudar, lavando los equipos, éramos campesinos".

Su historia laboral comienza en la empresa Petrosar en Sarmiento, Chubut, en el año 1972 dónde trabajó durante 10 años con un turno de 8 hs diarias. En el año 1982 llegó a Pico Truncado e ingresó en Piedra Clavada haciendo turnos de 8 horas diarias. 5 años más tarde, en 1987, tras un cambio de empresa, empezó en Came, donde cumplía 12 horas diarias durante un período de 10 meses. Francisco comenta: "Toda la vida trabaje 8 u 9 horas, siempre fue "normal" digamos. En los 90 cambiamos a 12 horas, se redujo personal. Yo protestaba, pero después me di cuenta que ganaba más, el básico siempre fue malo, no es que nos hayan aumentado mucho tampoco. No se veía mucha diferencia. Que nos den 12 horas era como un privilegio porque había muchos despidos en esa época. Las empresas asignaban quienes hacían esas horas porque el Gremio no quería saber nada con hacer 12 horas. A mí me la dieron porque un ingeniero me hablo y por ser mecánico de motores. Las 12 horas eran rotativas y cobrábamos fuera de convenio, fuera del gremio, nos daban más beneficios como, por ejemplo, en vales de viandas, pasajes, etc.

Las empresas eran inestables, los empleados migraban de empresa en empresa. Francisco recibió una oferta en Delia, trabajando 8 horas, pero pagadas como 12, ya que tenía otra categoría al tener más experiencia.

Como se mencionó anteriormente, las décadas del 70 y 80 en Santa Cruz estuvieron marcadas por conflictos sociales cuyo desenlace se produjo en la década del 90. Conflictos en relación con el trabajo, los piquetes por reclamos salariales, protestas sociales y organizaciones. Durante la dictadura militar de 1976, los gerentes empresariales estaban en conflicto con las fuerzas armadas, resultaban presos, generando una inestabilidad en las empresas petroleras. "Los policías estaban. El gerente estaba preso. Cayeron varios en la época de los militares. Había base militar, había que portarse bien".

Esta configuración laboral trajo consigo el despido y la reorganización de los trabajadores ante la inminente crisis del Gobierno de Carlos Saúl Menem. En Santa Cruz, el gobierno estaba a cargo de Néstor Kirchner, quien asumió en diciembre de 1991. La situación económica financiera en la provincia presentaba dificultades, poseía un déficit fiscal de 1.200 millones de dólares por lo que tuvo que declarar la emergencia económica. Las empresas no tenían estabilidad, eran configuradas y construidas por otras.

Agustín Salvia en "La Patagonia de los noventa: sectores que ganan, sociedades que pierden" menciona: 
"A la crisis por agotamiento productivo de muchas de las economías regionales se le suma hoy el impacto de las políticas de ajuste, los procesos de integración regional y las medidas de apertura externa y desregulación de los mercados. En ese contexto, también surgen nuevos negocios privados y se reactivan los procesos de acumulación y concentración del capital" (Salvia, 1999. Pág. 2).

Eduardo Basualdo (2002) en: "El proceso de privatización en la Argentina: la renegociación con las empresas privadas" también hace mención a la crisis de los noventa en relación con el accionar del sector político, dando continuidad a ciertas medidas que atribuían a las tomadas en el periodo del último gobierno militar:
“(...) Profundización de un modelo de acumulación cuyos denominadores comunes son la desindustrialización ligada a la crisis de las pequeñas y medianas empresas, el predominio de la valorización del capital, la centralización del capital, la concentración de la producción1 y el ingreso, la desocupación y la precarización de las condiciones laborales de los trabajadores, y la exclusión de un número creciente de individuos”. (Pág. 8)

Orietta Favaro (1998) en "La privatización de Yacimientos Petrolíferos Fiscales. Efectos en áreas petroleras de provincias" aborda en su estudio la crisis de los noventa y expresa que "El Estado favoreció el crecimiento de algunos capitales a los que subsidió, debilitó las empresas públicas y restringió los recursos destinados a los gastos sociales" (pág. 1).

La privatización de Yacimientos Petrolíferos Fiscales S. A. (YPF S. A.) en el año 1992, provocó en consecuencia que muchos trabajadores se quedaran sin trabajo y no pudieron subsistir a la crisis durante el Gobierno de Carlos Saúl Menem. Esto se debió, en parte, a la restructuración dentro de YPF S.A, incorporando trabajadores menores de 25 años con, entre otras características, un nivel de escolarización más elevada que incluya el idioma inglés (Favoro, 1998). De esta manera, esos empleados afectados se sumaron a las municipalidades, como en el caso de Pico Truncado y Caleta Olivia. La crisis petrolera y su ola de despidos marcó profundamente el panorama de la región obligando al diseño de políticas alternativas que permita sostener e impulsar el área productiva. De esta manera, ex trabajadores petroleros, empezaron a contribuir en la administración pública. "Muchos de los que trabajaban conmigo entraron a la Municipalidad o se fueron a Caleta Olivia a trabajar".

Agustín Salvia hace mención de ello:

"La administración pública municipal se convirtió en un importante sector de refugio ocupacional para desocupados y jóvenes en búsqueda de su primer trabajo. La situación también generó un importante crecimiento de actividades comerciales y de servicios informales, basadas en el autoempleo y la ayuda familiar no remunerada. Pero a pesar de estas estrategias de precarización, la tasa de desocupación regional alcanzó en 1993-94 el 16,3\% de la población económicamente activa" (Salvia, 1999. Pág. 19).

Esto produjo una desestabilización de la organización social entre los trabajadores. Comodoro Rivadavia, ciudad ubicada a $70 \mathrm{~km}$ de Caleta Olivia, no estaba exenta de la crisis laboral petrolera. Tal y como menciona Daniel Cabral Marques en "Lecturas en torno a YPF y al trabajo petrolero estatal en la cuenca del golfo San Jorge: diferentes miradas en una perspectiva de cien años": 
"Esta adscripción a la administración municipal que modificaba estructuralmente la situación de los pobladores de la zona norte de la ciudad se sustentaba en la necesidad de legitimar una nueva forma de ciudadanía social que rompía con la dinámica por la que se rigieron históricamente las empresas petroleras. A partir del nuevo estado de cosas que suponía la extensión del tejido municipal, la vieja figura de obrero o empleado petrolero que por su sola pertenencia laboral tenía asegurados los recursos necesarios para el mantenimiento de la vida cotidiana, comenzó a perder vigencia, instalándose progresivamente una racionalidad distinta, construida sobre la lógica del vecino contribuyente". (Cabral Marques, El libro del trabajo petrolero, 2019. pág. 56).

Las empresas fundadas en el ámbito privado no sufrieron los ajustes de este acontecimiento, por lo que los trabajadores privados tampoco vieron amenazados sus puestos de trabajo. Eduardo Basualdo (2002) en: "El proceso de privatización en la Argentina: la renegociación con las empresas privadas" menciona:

"Las empresas privatizadas han venido gozando de una multiplicidad de privilegios, desconocidos para los restantes agentes económicos que operan en el país. (...) De allí que no resulte casual que durante la década de los años noventa, la rentabilidad media del conjunto de las empresas privatizadas fuera entre siete u ocho veces superior a las de, incluso, el resto de las mayores firmas del país. (pág.4)”.

En las demás empresas las jornadas laborales se veían afectadas por la sobre exigencia. No se trabajaba los fines de semana porque no se les pagaba. Los trabajadores respondían mediante el paro. "Yo lucho por los míos". Los sueldos eran más bajos, muchas empresas quebraron al no solucionar los contratos laborales y por la mala administración interna, como el caso de Delia en Pico Truncado. Muchos de sus trabajadores migraron a empresas en la localidad de Las Heras. Las empresas que tenían convenio con YPF, como Quintana, empleaban por seis meses. Al finalizar los contratos, dependiendo el rendimiento laboral, calificaban como "personas no gratas", perjudicando el futuro laboral de sus trabajadores. A muchos de ellos les costó encontrar trabajo ante la crisis. Francisco Jaramillo fue una de ellas. Las mayorías de las familias contaban con una entrada económica, la del padre de familia, éste, al estar desempleado por periodos de 4 a 5 meses, buscaba otras alternativas para subsistir, como los comedores sociales. "Siempre había alguien que ayudaba, había una mujer que ayudaba a todos, le pedíamos fiado muchas veces, era chilena". En este sentido, aparece un concepto relevante en épocas de crisis económica, la solidaridad. Dicho concepto es considerado como sinónimo de proporcionar y de contribuir a reducir las desigualdades sociales entre sujetos, grupos, sociedades. Desde una perspectiva sociológica, la solidaridad se plantea como una acción ética y moral que los sujetos sociales potencian cuando se presentan situaciones de desigualdad y vulnerabilidad de los derechos humanos, creando así un sistema de colaboración entre las partes. En el caso del contexto desfavorable que sufrieron los ex trabajadores petroleros al ser despedidos, esta forma de cooperación y relación entre ellos, contribuyó a que las familias pudieran subsistir, como en el caso de la familia de Francisco Jaramillo, teniendo en cuenta que el único sostén familiar era él.

Surgían empresas que tenían convenios con otras, como San Cayetano, donde los ingenieros contrataban mediante una prueba realizada en la localidad de Comodoro Rivadavia, Chubut. Pero muchos trabajadores no lograban entrar por la mala reputación que se generó en empresas anteriores, como Delia. Estas empresas quebraron al poco tiempo por considerarse 
"cooperativas" y no recibir ayudas de empresas como YPF. Esta desestabilidad de las empresas hace surgir a otras, muchos eran ex trabajadores e ingenieros de YPF, sin embargo, para subsistir, debían tener una jornada laboral de 12 horas, había irregularidades en los contratos. Empresas como Metrape y Cove buscaban trabajadores sin estudios para trabajar con los neumáticos y limpieza de motores. Algunos tenían experiencia laboral al haber trabajado en otras empresas, otros no, por lo que "había tenido que voluntad de trabajar".

Los paros producidos por estas inestabilidades de las empresas petroleras y sus empleados producían desconfianza, Francisco afirma que "los delegados estaban vendidos al no hacer paro". Los delegados de los sindicatos no hacían paro, los trabajadores la pasábamos mal porque el sindicato iba en contra de los derechos de los trabajadores".

La informalidad del trabajo en la provincia se relaciona con el surgimiento de nuevas empresas. El trabajo informal, el oficio como una forma de subsistir pasa a considerarse trabajo formal bajo las empresas privadas. La crisis económica puso de manifiesto que los oficios eran compatibles solo en el sector petrolero en primera instancia. Jaramillo solo trabajó en empresas de esas índoles. Los trabajadores despedidos tuvieron que aprender otros oficios para entrar de nuevo al sistema laboral. Algunos con ayuda de sus pares.

Los trabajadores petroleros en la década del 90 demostraron ser un grupo social determinado por la construcción de un lazo afectivo entre los pares. Muchos de ellos se organizaban para enfrentar la discriminación de los salarios y los horarios laborales no remunerados. Los paros sindicales eran organizados y los trabajadores se diferenciaban de los gerentes. Ellos pertenecían a un grupo. De igual manera, las familias tenían un rol muy importante y constituye uno de los aspectos que caracterizan las dimensiones sociales de la década. Las familias de los extrabajadores petroleros estaban conformadas antes de migrar a la provincia. Muchos de ellos arribaban solos y una vez estabilizados, buscaban a su familia. Los hombres, en su mayoría, y como Jaramillo así lo indicaba, eran los únicos que trabajan en el círculo familiar. Las mujeres eran amas de casa. Por ende, la crisis que atravesó la década potenció la desigualdad social. Había comedores y gente que ayudaba a las familias que no tenían entrada económica.

\section{METODOLOGÍA}

La carrera Licenciatura en Comunicación Audiovisual proporcionó herramientas de investigación prácticas como la cámara como forma de registro, de esta manera la cámara y el objeto de estudio son dos sujetos sociales activos. El registro audiovisual contribuye la relación de audio con imagen. La imagen se define por el plano, el encuadre y la fotografía. Las entrevistas en el área audiovisual están determinadas por un plano medio del protagonista, definiendo su rostro, de esta manera se involucra los gestos y los movimientos del entrevistado. El sonido en este caso fue el testimonio de Francisco Jaramillo. Como se suele trabajar en formato documental, la cámara como forma de registro en investigación debe tener la mínima "manipulación posible". La cámara es una extensión del equipo de investigación que trata de registrar lo que el investigador no puede en este caso. Las entrevistas registradas por la cámara equivalen a una nota de campo. Esta metodología de trabajo se aplicó para poder reforzar y contribuir a la técnica audiovisual en el área de investigación como se estuvo trabajando en el Gabinete de Experiencias Pedagógicas: Pensamiento y Habla en la UNPA UACO.

La utilización de las herramientas audiovisuales se complementa con el diseño metodológico siguiente:

- La identificación de un sujeto social clave. En este caso, se determinó trabajar con el testimonio de Francisco Jaramillo de la ciudad de Pico Truncado. Él es uno de los agentes 
principales que reúne características que aportan a la dinámica de Santa Cruz de la década del 90. Jaramillo es jubilado del petróleo y parte del proceso de migración y de población de Santa Cruz en la década del 80, atendiendo como ex empleado de empresas petroleras como mecánico de motores, un oficio importante en esa época.

- Relevamiento de fuentes bibliográficas que permitan complementar con el estudio del campo de los 90. De esta manera, la lectura de autores como Agustín Salvia e investigadores de la UNPA y la UNSJB permitieron conocer aspectos tales como la dinámica empleada en los orígenes del petróleo en la zona de Comodoro Rivadavia y el noroeste de Santa Cruz, permitiendo acceder al contexto anterior y de mediados de los años 90.

- Entrevista e implementación del área audiovisual. Esta fue la técnica fundamental a implementar, ya que de esta manera y en el problema investigado, se accede al registro del testimonio de Francisco Jaramillo. La herramienta audiovisual de respaldo de investigación fue la cámara y corresponde a uno de los ejes fundamentales de la Licenciatura en Comunicación Audiovisual. En este caso, fue un respaldo influente. La voz y la imagen representan otro punto de análisis de un discurso. La posición de los agentes, el registro consensuado por el agente a la hora de ser grabado contribuye a tener material físico y de archivo para próximas investigaciones.

De esta manera, las herramientas contribuyeron a abordar la trayectoria laboral de Francisco Jaramillo y su forma de aprendizaje a través del oficio para su inserción en la actividad laboral petrolera.

\section{Reflexiones. Discusiones.}

Los aportes del testimonio de Francisco Jaramillo permitieron comprender e identificar formas de institucionalizar los oficios en las empresas, entre otras categorías que, en relación con lo investigado por Agustín Salvia en "La Patagonia de los 90, sectores que ganan, sectores que pierden", son propicias de nombrar. Por ejemplo, Salvia menciona (1999):

- "La producción económica se concentraba en pocas ramas. La estructura económicoocupacional de esta área indicaba un alto grado de división del trabajo social y por ende de desarrollo de las fuerzas productivas" (Salvia, 1999, pág. 7). Francisco Jaramillo menciona que aprendió a limpiar motores y que no había mucha gente que sabía hacerlo, por lo que él fue uno de los "privilegiados". F. Jaramillo: "Sabia de mecánica, tenía buenas referencias, era joven con energía y buena salud, tenía ganas de trabajar, de progresar y crecer". "Aprendí hablando con los ingenieros, empecé con la voluntad de ayudar, lavando los equipos, éramos campesinos".

- Proceso de restructuración. Salvia menciona que este proceso impulsó el quiebre de empresas o de una reorganización que provoco desempleo a través de despidos y renuncias. Esto se relaciona con lo mencionado por F. Jaramillo. El menciona que la crisis afecta el número de horas trabajadas, pasadas de 8 a 12 con una mínima diferencia en el salario, por lo que algunos de sus compañeros renunciaban. Otros eran despedidos. Algunas empresas se fusionaban con otras. F. Jaramillo: "Había mala administración interna, como en la empresa Delia por ejemplo, por eso algunos se iban a Las Heras a trabajar, los tenían a las vueltas entonces se iban, si te contrataban después, era por 6 meses y después veías como seguías". Salvia apunta también que esto conllevo a que muchos trabajadores precarizados cubran demandas transitorias y movimientos espaciales para conseguir algún empleo.

- Unidad sindical y organización gremial. Francisco Jaramillo recuerda que como trabajadores eran compañeros y unidos. "Nosotros somos un equipo, nos ayudábamos 
entre todos para aprender". "Trabajamos los fines de semanas y no nos pagaban esos días, entonces hacíamos paro, yo luchaba por los míos". Sin embargo, al comenzar la crisis de la privatización, la desconfianza se empezó a notar entre la estructura empresarial: "Los delegados estaban vendidos al no hacer paro". Los delegados de los sindicatos no hacían paro, los trabajadores la pasábamos mal porque el sindicato iba en contra de los derechos de los trabajadores". Salvia menciona que, a raíz de estos quiebres en las relaciones laborales, los trabajadores también mostraban competencia entre ellos.

Jaramillo menciona que, al principio se veía como algo normal, pero después luchaban por conseguir o mantenerse en el lugar, algunos dejaban de hacer paro por miedo.

En relación con lo mencionado anteriormente, se destaca lo mencionado por Salvia, lo cual resume y aporta a contextualizar la dinámica de los 90 en Santa Cruz:

"Fenómenos como la acumulación flexible, la informalidad económica, la precariedad laboral, la desocupación y otras formas de subutilización de fuerza de trabajo se constituyen componentes comunes, tanto en mercados regionales dinámicos como tradicionales, en economías centrales como en zonas atrasadas o periféricas, en complejos tecnológicos como en distritos industriales o mineros en crisis (Salvia, 1999, pág. 5).

Para finalizar, en relación con el análisis del testimonio de Francisco Jaramillo se considera válido señalar y recuperar lo expresado por Maria Rosa Cicciari (2001) sobre las trayectorias laborales: “(...) designan las distintas ocupaciones de los actores, sus antecedentes y experiencia en el mundo del trabajo y, a la vez, hacen referencia a la posibilidad de diseñar proyectos más o menos deseables". A su vez, la misma investigadora hace referencia a que:

"Otros estudios rescatan el avance en la búsqueda del sentido y la direccionalidad de los procesos de cambio estructural, a través de la reconstrucción de las trayectorias laborales de conjuntos poblacionales específicos. (...) Esta propuesta rescata la posibilidad de indagar sobre la existencia de ciertas trayectorias donde lo social se expresa a través de las historias individuales; a partir de la reconstrucción del acontecer de la vida laboral que revela su participación y vinculación con los procesos de carácter social” (pág. 3)

En este trabajo, el testimonio de Jaramillo posibilitó la reconstrucción de su propia trayectoria laboral y las marcas que ésta imprimió en su vida personal futura.

\section{CONCLUSIONES}

El testimonio de Francisco Jaramillo es un testimonio que no debe perderse en el tiempo al haber pasado más de 30 años. La utilización de la cámara como herramienta de investigación ayuda a que esto no se pierda y es una forma de registro físico que contribuye a establecer otro nivel de relación entre sujetos de investigación. Muchos de los ex trabajadores petroleros que atravesaron las décadas del 80 y 90 en Santa Cruz, actualmente son jubilados, otros siguieron trabajando, aprendiendo y enseñando oficios. Por ello, los relatos olvidados en el tiempo de estos trabajadores son los que más información poseen. Francisco Jaramillo, es un padre de familia que quiso contar su experiencia, su trayectoria y su propia historia laboral que tuvo lugar recorriendo el Norte de la provincia entre ciudades como Las Heras, Caleta Olivia y finalmente Pico Truncado, lugar en el que vive hace cuatro décadas. ¿Qué impulsó parte de esta dinámica social en Santa Cruz? Gracias a su testimonio, se comprendió el 
progreso y la voluntad de crecer internamente como sujeto de derecho. Esto, inspiró a la creación de nuevas herramientas de aprendizaje y de conocimiento. Los trabajadores aprendían al escuchar al otro como par, y experimentando empíricamente el oficio. Las prácticas estaban determinadas por el trabajo forzado, muchos de ellos no tenían estabilidad. Las crisis impulsaron una nueva dinámica laboral y social. La década del 90 constituyó a una formación y un proceso de aprendizaje vinculada a la categoría del trabajo. Las empresas buscaban la estabilidad económica igual que sus trabajadores. Las ciudades estabilidad social, se redistribuyó la población, se urbanizaron los pueblos, en palabras de Francisco Jaramillo: "algunos quedaron como si el tiempo no hubiera pasado". Es por ello que el aporte de un ex trabajador petrolero que trabajo durante 42 años, influye y aporta en parte a esta dinámica social en la década del 90.

\section{BIBLIOGRAFÍA}

BASUALDO, E. (2002). El proceso de privatización en la Argentina: la renegociación con las empresas privadas, Flacso.

CICCIARI, M. R. (2001). Trayectorias laborales en espacios sociales urbanos afectados por el proceso de reestructuración productiva. Estudio de caso: Comodoro Rivadavia en los años '90". Proyecto de Investigación "Efectos y perspectivas de desarrollo en el litoral atlántico de la Patagonia Austral. Sectores que ganan, sociedades que pierden en el contexto de cambio estructural de los años '90”. UNPA -UACO.

FAVARO, O. (1998). La privatización de Yacimientos Petrolíferos Fiscales. Efectos en áreas petroleras de provincias. Revista de Historia - Facultad de Humanidades

MARQUES, D. (2019). Lecturas en torno a YPF y al trabajo petrolero estatal en la cuenca del Golfo San Jorge: Diferentes miradas en una perspectiva de cien años. En: Gabriel Carrizo (Comp.) El mundo del trabajo petrolero en la cuenca del Golfo San Jorge. (pp. 30-64). Río Gallegos, Argentina: UNPA.

REBOllO J., ARDÉVOL E., CANAL G., GUEVARA, A. (2008). El medio audiovisual como herramienta de investigación social. Barcelona. CIDOB edicions.

ROZAS, D., NAVARRO, M. F. (2019). Informe final PI 29/D079 “Cartografías de los 90. Cultura y política en Santa Cruz. UNPA.

SALVIA, A. (1999). La Patagonia de los noventa: Sectores que ganan, Sociedades que pierden. Buenos Aires: La Colmena. 